\title{
Impact on Sexual Function of Endoscopic Enucleation vs Transurethral Resection of the Prostate for Lower Urinary Tract Symptoms Due to Benign Prostatic Hyperplasia: A Systematic Review and Meta-Analysis
}

\author{
Yuqing Liu, MD, ${ }^{1, *}$ Yinchu Cheng, PhD, ,,* Lin Zhuo, $\mathrm{PhD},{ }^{3}$ Ke Liu, MD, ${ }^{1}$ Chunlei Xiao, MD, \\ Rongsheng Zhao, $\mathrm{PhD}^{2}$, Jian $\mathrm{Lu}, \mathrm{MD}^{1}$, and Lulin $\mathrm{Ma}, \mathrm{MD}^{1}$
}

\begin{abstract}
Introduction: Although the transurethral resection of the prostate (TURP) has been regarded as the gold standard surgical option for lower urinary tract symptoms due to benign prostatic hyperplasia, the endoscopic enucleation of the prostate (EEP) with novel techniques is also recommended. However, whether there are differences on male sexual functions after these two procedures is still controversial. We performed this metaanalysis to investigate the impact on erectile function (EF) and ejaculation of EEP and TURP, and to explore the potential superiority between these two approaches.

Materials and Methods: Literature search was conducted on Medline, Embase, and the Cochrane library, to obtain all relevant randomized controlled trials published before December 13, 2018. Outcome data were pooled and analyzed with Review Manager 5.3 to compare the International Index of Erectile Function 5 (IIEF5) scores and retrograde ejaculation (RE) rates from short- to long-term follow-ups.

Results: Ten articles with 1435 patients were included. EEP group showed higher IIEF-5 scores than TURP group with mean difference $(\mathrm{MD})=1.00$, confidence interval $(95 \% \mathrm{CI})$ : $0.95-1.05$ at 48 months and $\mathrm{MD}=1.08$, 95\% CI: $1.03-1.13$ at $\geq 60$ months, respectively. In subgroup analysis, the holmium laser enucleation of the prostate showed significantly better IIEF scores than TURP group at 48 months ( $\mathrm{MD}=1.00$, 95\% CI: $0.95-$ 1.05 ) and $\geq 72$ months ( $M D=1.08,95 \% \mathrm{CI}: 1.03-1.13$ ). Additionally, no significant difference in RE rate was found between EEP and TURP (risk ratio $=1.02,95 \%$ CI: $0.85-1.23$ ).

Conclusions: Compared with TURP, EEP may produce a more durable benefit for postoperative preservation of EF at long-term stage.

Keywords: benign prostatic hyperplasia, endoscopic surgical procedure, transurethral resection of prostate, penile erection, ejaculation, meta-analysis

\section{Introduction}

$\mathbf{L}$ OWER URINARY TRACT SYMPTOMS (LUTS) due to benign prostatic hyperplasia (BPH) is one of the most common clinical complaints in aged men, with an estimated rate of $10 \%$ in their 50s and of almost $90 \%$ in men over 80 years of age. ${ }^{1}$ Beyond the benign definition, BPH can become more serious because of its various complications during its longterm course, and can cause sexual dysfunction, including decreased libido, erectile dysfunction (ED), or decreased sexual satisfaction. ${ }^{2}$ Although a life-time medical therapy is suggested as first-line management for LUTS/BPH, there is

still a high discontinuation rate that is most likely attributed to both drug side effects on sexual functions and patients' high expectations. ${ }^{3}$

The surgical approach remains one of the most effective managements of certain BPH cases with severe and complicated conditions or LUTS refractory medical therapy. ${ }^{4}$ Despite the fact that the transurethral resection of the prostate (TURP) has been regarded as the gold standard surgical option for LUTS due to BPH, the high rate of intraoperative or postoperative morbidities associated with TURP motivated investigators to search for modifications or alternative procedures. ${ }^{5}$ Plasmakinetic resection of prostate (PKRP), also
\end{abstract}

Departments of ${ }^{1}$ Urology and ${ }^{2}$ Pharmacy, Peking University Third Hospital, Beijing, China.

${ }^{3}$ Research Center of Clinical Epidemiology, Peking University Third Hospital, Beijing, China.

*These authors contributed equally to this work. 
known as bipolar TURP (B-TURP), is a newly developed technology as the modification of standard TURP with monopolar electrocautery system, and demonstrates comparable short-term and long-term efficacy with TURP. ${ }^{6}$

With the evolution of novel technologies in the last two decades, a wide range of innovative endoscopic procedures have challenged TURP supremacy. During this period, the leading principle of the endoscopic management for patients with LUTS/BPH was converted from excision of small tissue chips and subsequent irrigation from bladder to en-bloc removal of the adenoma from the surgical capsule and subsequent morcellation. ${ }^{7}$ Recommended by the updated European Association of Urology (EAU) guidelines, endoscopic enucleation of the prostate (EEP) has been considered as an advanced form of transurethral prostatectomy for any prostate size with equivalent outcomes to open prostatectomy, and is an increasingly popular option for men with LUTS due to $\mathrm{BPH}^{4}$

The emergence of additional energy sources brought about a series of burgeoning modalities of EEP. Several sorts of contemporary lasers, such as holmium and thulium lasers, have been used for enucleation of prostatic adenomas of any size, known as holmium laser enucleation of the prostate (HoLEP), ${ }^{8}$ thulium vapoenucleation of the prostate (ThuVEP), or thulium laser enucleation of the prostate (ThuLEP). ${ }^{9}$ Besides these, there has also been resurgent interest in the use of bipolar electrosurgery, and the plasmakinetic enucleation of the prostate (PKEP) has been proved to be safe and technically feasible. ${ }^{10}$

There were several meta-analyses on the direct comparison of the efficacy and safety of transurethral enucleation and resection of prostate for LUTS due to BPH, but few of them focused the treatment impact on erectile function (EF) or ejaculation function (EJF) ${ }^{11-13}$ One such systematic review and network meta-analysis compared the International Index of Erectile Function 5 (IIEF-5) scores after different surgical treatments for LUTS/BPH, but it did not evaluate EJF, which is also an important component of overall sexual function. ${ }^{14}$ In another earlier meta-analysis, including comparison of the impact on EF or EJF between TURP and holmium laser treatment, the recently developed methods of EEP such as thulium laser enucleation or PKEP were not included. ${ }^{15}$

Therefore, it is necessary to carry out a meta-analysis to evaluate the impact of endoscopic enucleation and resection of the prostate on male sexual functions. Based on a systematic review of randomized controlled trials (RCTs), the current study addressed this need by comparing the effect of TURP, including modified methods like PKRP and updated procedures of EEP, including laser enucleation as well as PKEP on EF or EJF.

\section{Materials and Methods}

We performed and reported this systematic review in accordance with the Preferred Reporting Items for Systematic Reviews and Meta-Analyses (PRISMA) Statement. ${ }^{16}$ The protocol of this systematic review was published on the PROSPERO register (www.crd.york.ac.uk/PROSPERO).

\section{Literature search}

We searched Medline, Embase, and the Cochrane library for published articles. ClinicalTrials.gov was also searched to identify potentially relevant studies. All searches were performed on December 13, 2018. Detailed search strategies are listed in Supplementary Data. We also examined the reference lists of retrieved articles and related reviews. Language was restricted to English.

\section{Eligibility criteria}

Studies were included if they met the following criteria: (1) study population with clear diagnoses of BPH or LUTS; (2) RCTs, which compare endoscopic enucleation and TURP; (3) at least one of the outcomes on sexual function was reported quantitatively, including EF (measured by IIEF-5 score) and retrograde ejaculation (RE). Duplicate articles, ongoing studies, and those without available results were excluded.

\section{Study selection and data extraction}

We screened the titles and abstracts of the articles identified from literature search and excluded clearly irrelevant ones according to the eligibility criteria described above. The remaining studies were reviewed in full text further for inclusion assessment. Information of each included trial was extracted using a predefined Excel form. Extracted data included study characteristics (author, publication year, setting), follow-up duration, sample size, intervention and control arms, participants' baseline characteristics (age, prostate volume, IIEF score), and outcome statistics. Two review authors (Y.L. and Y.C.) independently selected study for inclusion and extracted data, any disagreements were resolved by discussion or by a third investigator (J.L.).

\section{Quality assessment}

The methodology quality of included studies were assessed independently by two review authors (Y.L. and Y.C.) using the Cochrane's "Risk of bias" assessment tool. ${ }^{17}$ The following seven items were adjudicated low, unclear, or high risk of bias: random sequence generation, allocation concealment, blinding of participants and personnel, blinding of outcome assessment, incomplete outcome data, selective reporting, and other bias. Since sexual function evaluated by IIEF-5 questionnaire is a subjective patient-reported outcome, we think that the blinding of participants and outcome assessment is important to avoid relevant bias. Therefore, studies without blinding were considered to have high risk of bias in the respective item. For the item of "other bias," studies funded by industry were judged to have high risk of bias. Any disagreements were resolved through discussion.

\section{Data analyses}

Risk ratios (RR) and mean differences (MDs) with confidence intervals (95\% CIs) were calculated for binary and continuous outcomes, respectively. Heterogeneity among studies were evaluated with Cochrane $Q$ test and the $I^{2}$ statistic. A fixed-effects analytical model was used to pool the results of studies with low or acceptable heterogeneity ( $p$ for $Q$ test $>0.10$ ). Subgroup analysis was conducted to explore between-study heterogeneity according to different interventions. We also performed a sensitivity analysis by excluding studies with at least one domain of adjudicative high risk of bias. We were unable to assess publication bias by funnel plot due to the scant number of included studies. All 
statistical analyses were conducted using Review Manager (RevMan) 5.3 (Copenhagen: The Nordic Cochrane Center, The Cochrane Collaboration, 2014).

\section{Results}

\section{Search results and study selection}

The literature selection flowchart is summarized in Figure 1. Database searching yielded a total of 415 references, including 13 unpublished studies from ClinicalTrials.gov. After abstract screening and full-text review, 10 eligible studies were included for final analysis, ${ }^{18-27}$ contributing a total sample size of 1435 patients. A summary of the characteristics of each included study is presented in Table 1. Seven of the 10 studies used HoLEP as intervention, 2 used PKEP, and 1 used ThuLEP. All of the studies used TURP/PKRP/bipolar-TURP as comparator. The minimum follow-up duration was 3 months, and the maximum was up to 92 months. Mean age of participants at baseline varied from around 64 to 72 years among the studies, and the patients had a mean baseline prostate volume from 56.7 to $113.8 \mathrm{cc}$
FIG. 1. Flow chart of literature search and study selection.
415 references identified from database search:

Medline, $N=141$

Embase, $N=196$

The Cochrane Library (including CENTRAL), $N=65$

ClinicalTrials.gov, $N=13$

142 duplicates

273 abstracts screened

220 abstracts excluded:

Reviews, editorials, letters, etc., $N=110$

Irrelevant patients, $N=5$

Not comparing enucleation surgeries with transurethral resection of the prostate, $N=38$

Observational study, not randomized study, single arm study, etc., $N=33$

Duplicates, $N=16$

Not in English, $N=1$

Results not available, $N=13$

Ongoing study, $N=4$
43 studies excluded:

Without extractable data on sexual function, $N=37$

Reviews, editorials, letters, etc., $N=1$

Not comparing enucleation surgeries with transurethral resection of the prostate, $N=1$

Duplicates, $N=2$

Not in English, $N=2$

10 studies included for final analysis 


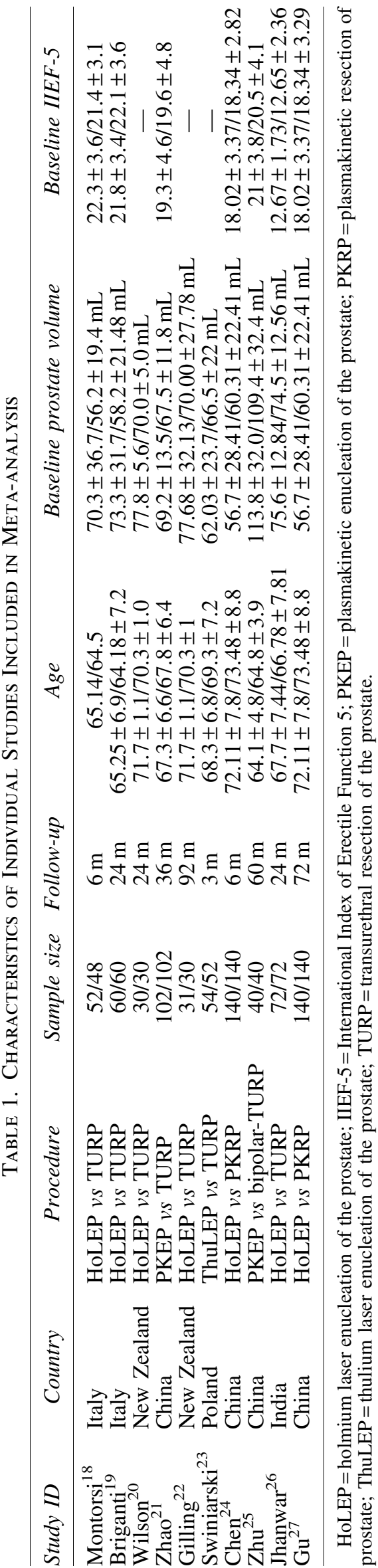

\section{Quality assessment}

The results of study quality assessment are shown in Figure 2 and Supplementary Fig. S1. Seven studies were judged to have low or unclear risk of bias in each domain, of which two ${ }^{24,27}$ had low risk of bias in all domains. With the absence of blinding, three studies ${ }^{21,22,25}$ might have a high risk of performance or detection bias.

\section{Sexual function}

Erectile function (IIEF-5). Eight studies reported results on EF measured using IIEF-5 at 6 to 92 months of follow-up, comparing transurethral enucleation $v s$ resection of the prostate. The forest plots are presented in Figure 3. The MD

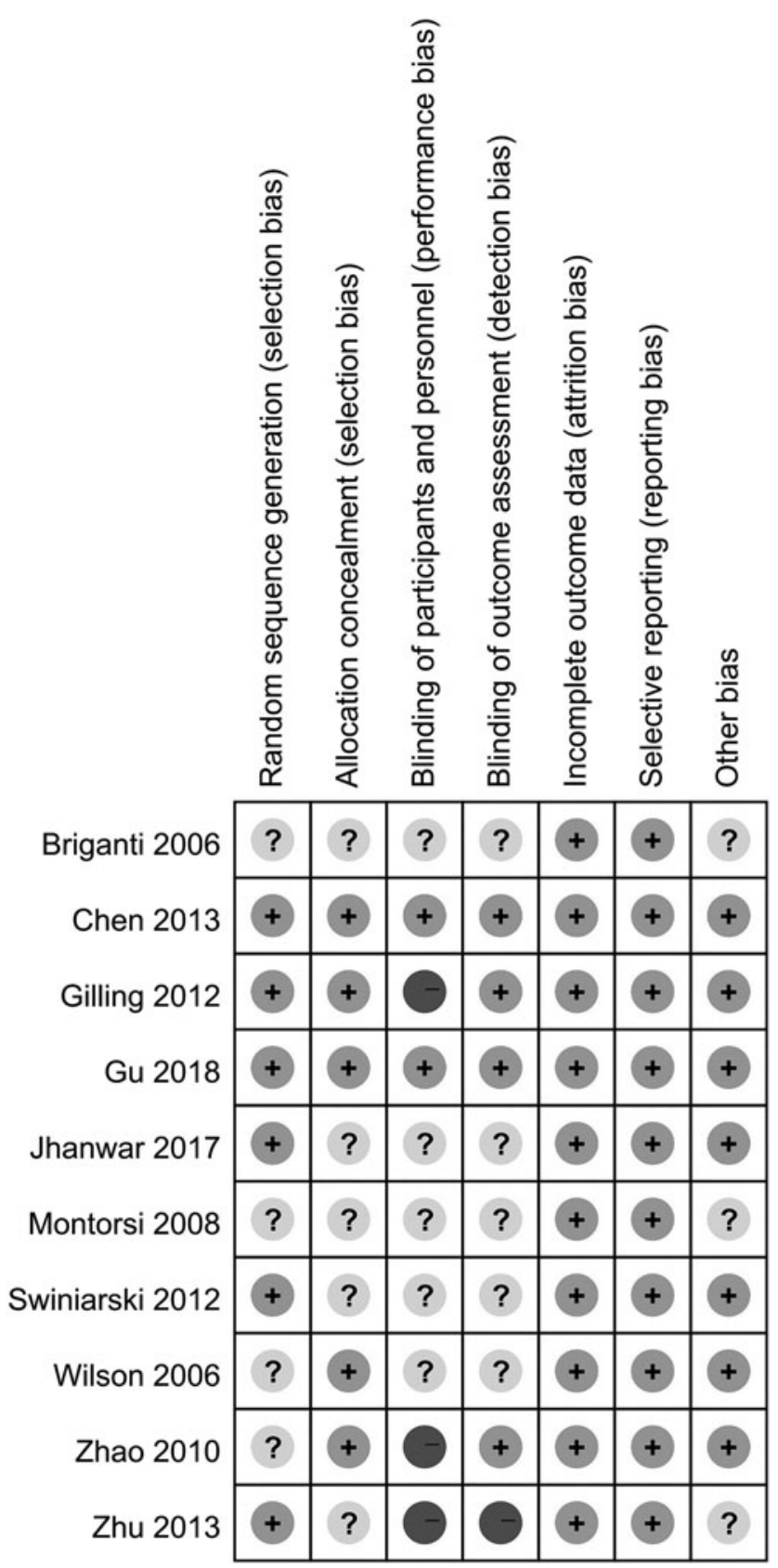

FIG. 2. Risk of bias summary: review authors' judgments about each risk of bias item for each included study. 


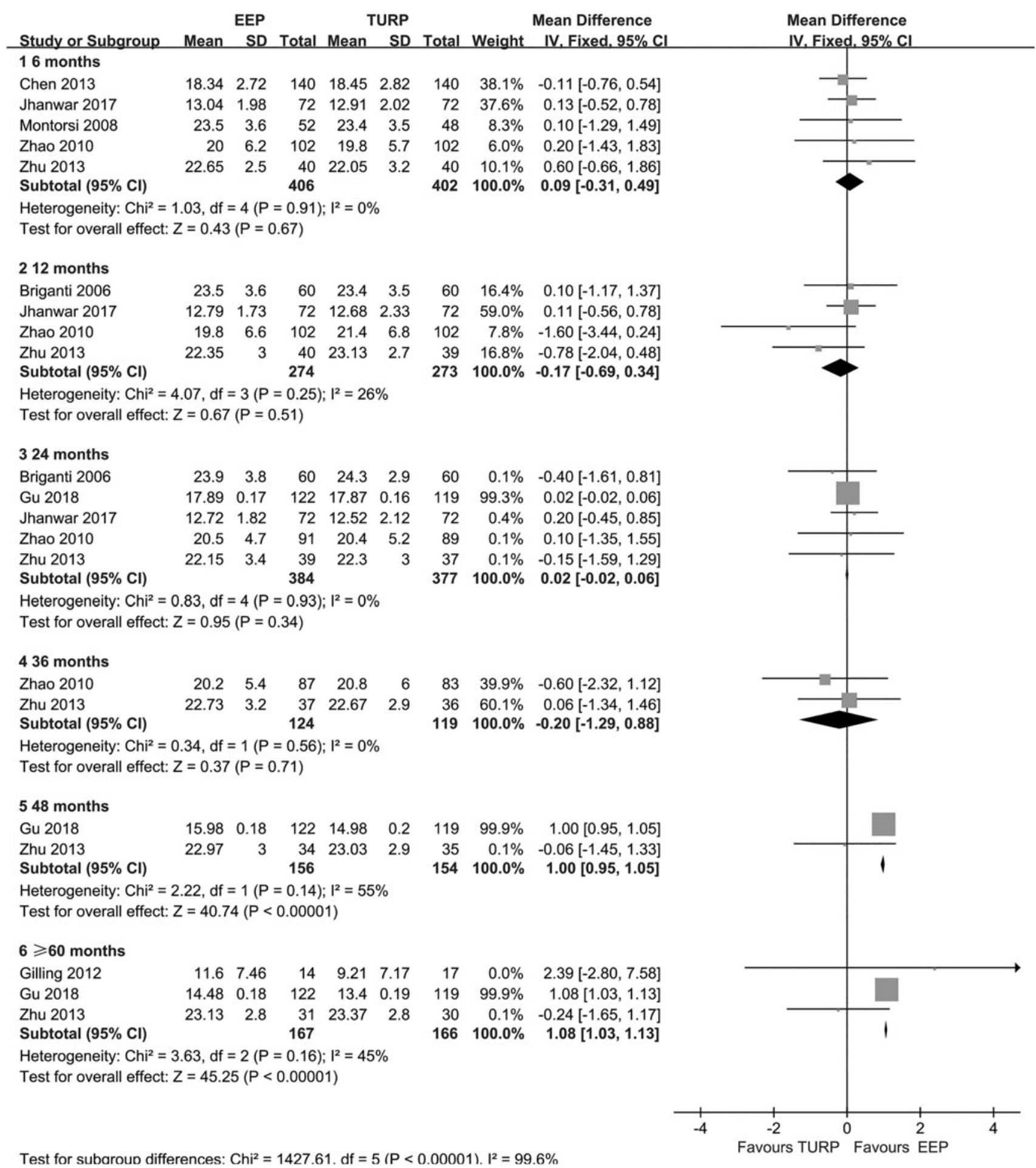

FIG. 3. Forest plots for IIEF-5 scores after EEP $v s$ TURP at 6, 12, 24, 36, 48, and over 60 months. IIEF-5=International Index of Erectile Function 5; EEP = endoscopic enucleation of the prostate; TURP = transurethral resection of the prostate.

of IIEF-5 score at month 6, 12, 24, and 36 was not statistically significant between EEP and TURP. However, EEP group started to show significantly higher IIEF-5 score than TURP group at longer follow-up time (48 months, $\mathrm{MD}=1.00,95 \%$ CI: $0.95-1.05 ; \geq 60$ months, $\mathrm{MD}=1.08,95 \% \mathrm{CI}: 1.03-1.13$ ).

Subgroup analysis was performed separately for HoLEP $v s$ TURP (Fig. 4) and PKEP vs TURP (Fig. 5). A similar trend, that is, significantly better IIEF score only after 48 months since surgery, was found in HoLEP group compared with TURP group (48 months, MD:1.00, 95\% CI: 0.95-1.05; $\geq 72$ months (MD:1.08, 95\% CI:1.03-1.13). Shorter term results were comparable between the two groups. One study ${ }^{19}$ also compared the frequency of patient-reported ED between HoLEP and TURP at 12 months (odds ratio [OR] $=0.91,95 \%$ 


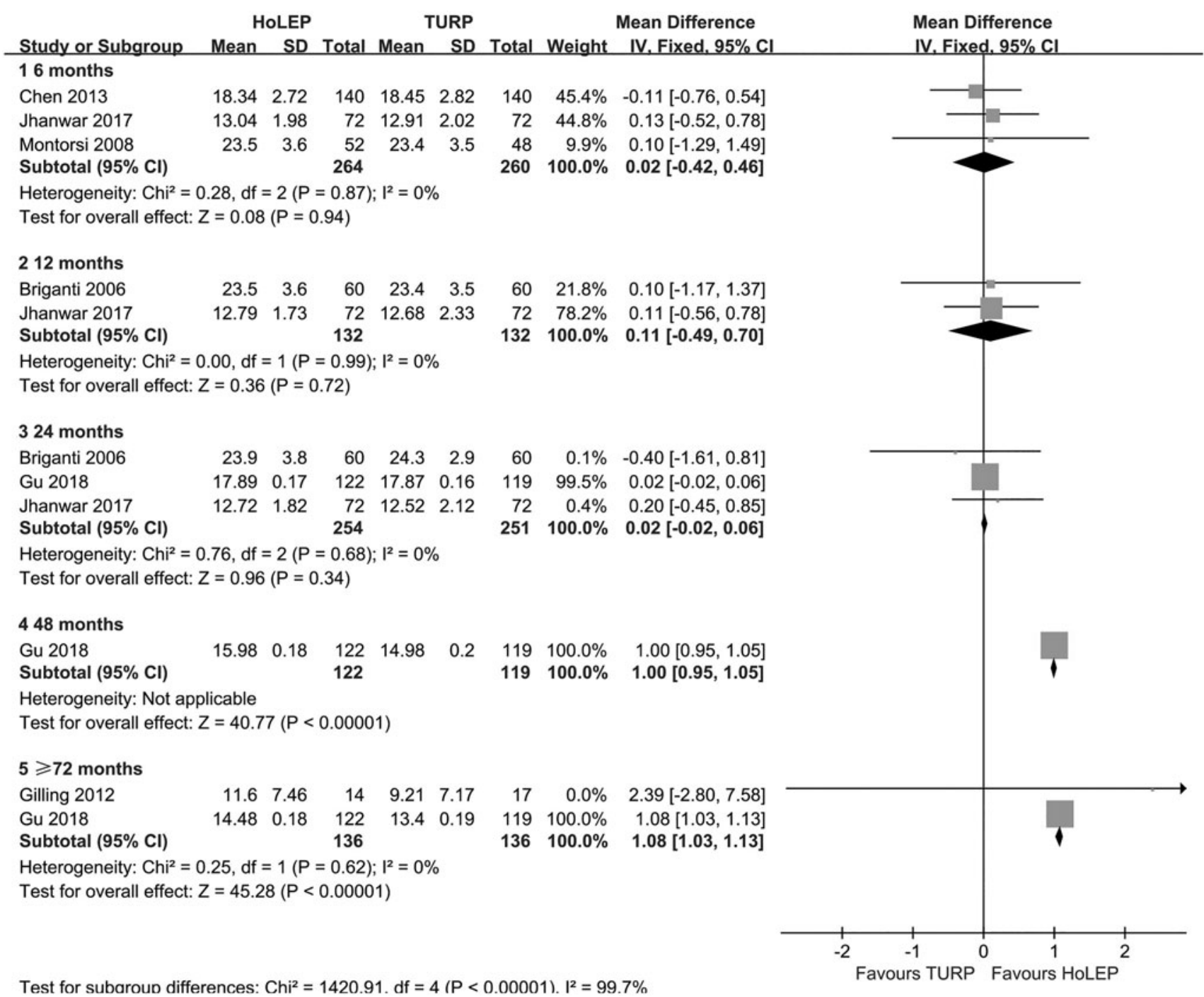

FIG. 4. Subgroup analysis for IIEF-5 scores after HoLEP $v s$ TURP at 6, 12, 24, 48, and over 72 months. HoLEP=holmium laser enucleation of the prostate.

CI: $0.39-2.14)$ and 24 months $(\mathrm{OR}=1.00$, 95\% CI: $0.42-$ 2.38), showing no significant difference as well. Data were somewhat limited, however, for PKEP $v s$ TURP. Based on two current available studies, ${ }^{21,25}$ there was no significant difference between IIEF-5 scores at all at shown measured time points from 6 to 60 months.

Retrograde ejaculation. Four studies reported the frequency of RE after EEP and TURP, one at month $3^{23}$ and three at month 24. ${ }^{19,20,27}$ As shown in the forest plot (Fig. 6), no significant difference in RE was found between EEP and TURP $(\mathrm{RR}=1.02,95 \% \mathrm{CI}: 0.85-1.23)$.

\section{Sensitivity analysis}

After excluding studies with at least one domain of adjudicative high risk of bias, ${ }^{21,22,25}$ results for EF between HoLEP and TURP did not change significantly (Supplementary Fig. S2), but no data for PKEP vs TURP remained available. Sensitivity analysis for RE was not performed because of a limited number of eligible studies.

\section{Discussion}

The ultimate aim of all therapy regimens for $\mathrm{BPH}$ is not only the relief of LUTS, but also the improvement of quality of life. ${ }^{1,2}$ Although surgical treatments for BPH have proven to be effective to relieve benign prostatic obstruction, the effects of invasive operations on postoperative sexual function are still under debate. ${ }^{11}$ There is a hypothesis that the postoperative improvement in LUTS will indirectly preserve sexual functions, especially $\mathrm{EF},{ }^{14}$ because the significant decreases in nocturia will lead to increases in oxygen supply to the corpus cavernosum, sexual desire, and penile erections. ${ }^{8}$ Besides this, the improvement in sexual function after surgery may be partially attributed to the cessation of preoperative medications. $^{19}$

Standing the test of time, TURP has remained the mainstay of procedure for BPH with significant clinical improvement in LUTS with considerable complication rates, ${ }^{6}$ including a discordant incidence of postoperative ED. ${ }^{19}$ There are generally accepted postulations that the heating effect of electrocautery causes the cavernous nerve and vascular injuries, ${ }^{14}$ 


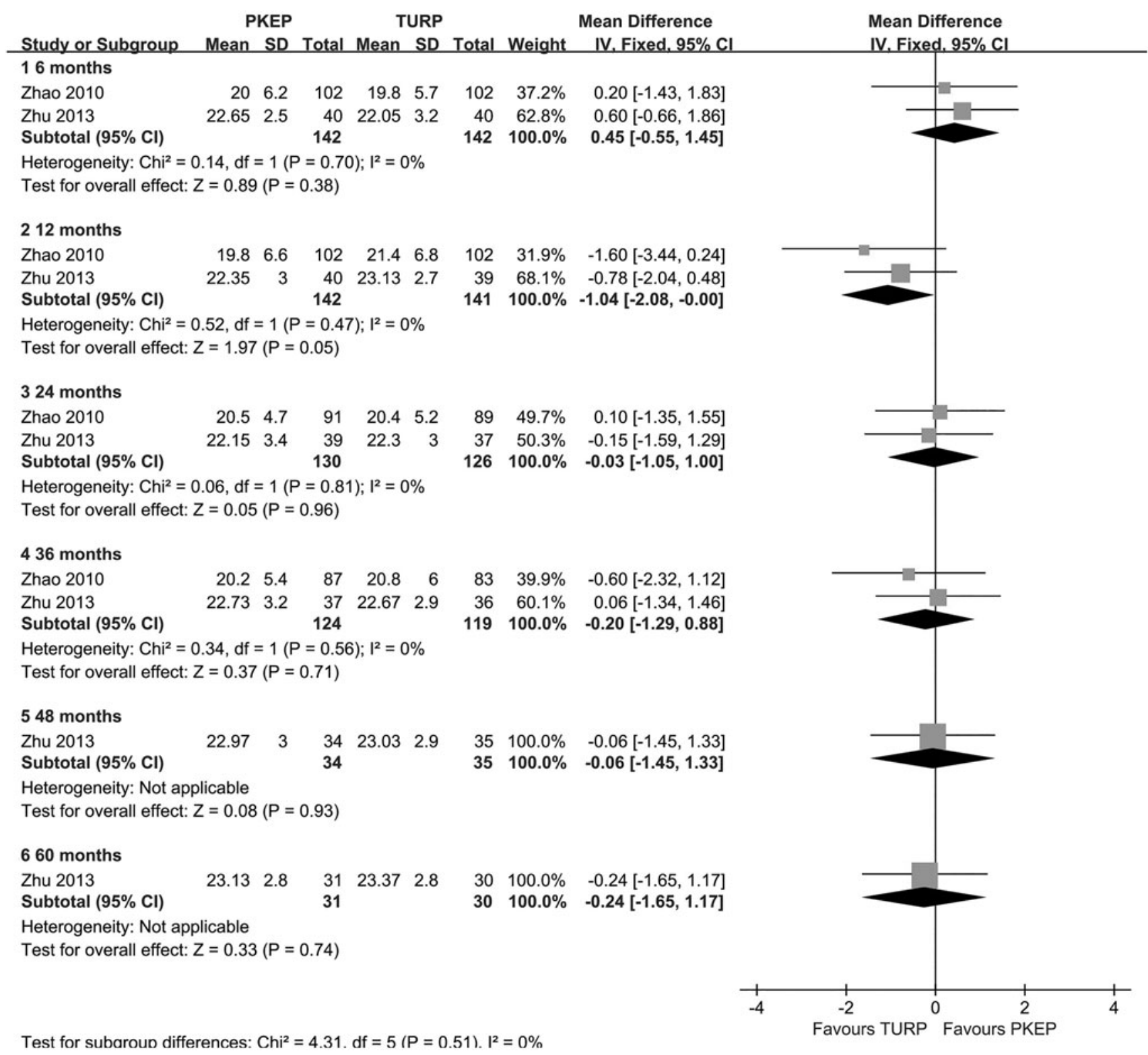

FIG. 5. Subgroup analysis for IIEF-5 scores after PKEP $v s$ TURP at $6,12,24,36,48$, and 60 months. PKEP=plasmakinetic enucleation of the prostate.

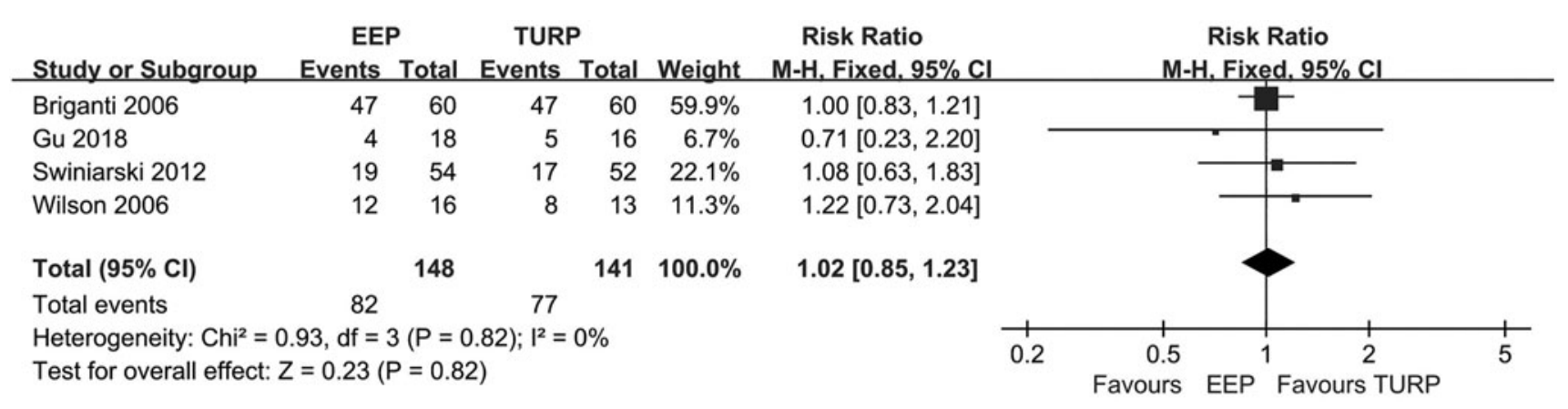

FIG. 6. Forest plot for retrograde ejaculation rates after EEP vs TURP. 
and the neuropraxia and emotional stress may lead to impotence after TURP. ${ }^{19}$

Both monopolar and bipolar TURP have been reported to be associated with a mild decline in postoperative IIEF-5 scores with no difference in long-term follow-up. ${ }^{28}$ However, it is necessary to take into consideration that the population investigated in these studies often already suffers from preoperative ED. In fact, only few studies specifically analyzed the incidence of de novo ED in a prospective fashion. In a retrospective study, although a 5.8\% incidence of a worsening EF after TURP was reported, there was a total of $16.2 \%$ of patients who reported a postoperative improvement of EF. ${ }^{29}$ For patients with normal baseline EF, a systematic review and meta-analysis showed that TURP improved the postoperative IIEF-5 scores in over 12 months of follow-up. ${ }^{14}$

In recent years, EEP has been developed and recognized as a viable treatment option due to reduced morbidity and shorter convalescence. ${ }^{11-13}$ The basic procedure of enucleation is removing adenoma of prostate along the surgical capsule plane with less heating effect and vascular injury, so EEP was considered of potential benefit to preserving the sexual function. ${ }^{8,10}$ Marginal and nonsignificant EF improvement was reported in the analyses of 12- and 24-month post-HoLEP IIEF scores, ${ }^{19,30}$ and in the 12-month follow-up evaluation after ThuVEP. 9

Despite this, not all patients with LUTS/BPH will benefit from EEP in terms of EF. Long-term sexual outcomes showed that the patients with more severe preoperative urinary symptoms and ED would be more likely to have a clinically meaningful EF improvement, which was attributed to a persistent amelioration of LUTS. ${ }^{31}$ For the patients without preoperative ED, a relatively small, but still significant, negative effect on erections in the early period after HoLEP was reported. ${ }^{8}$ Thus, it is still controversial for the impact on sexual functions between enucleation and resection of prostate.

There were a number of RCTs that have directly compared postoperative sexual functions between enucleation and resection of prostate for LUTS/BPH. In this analysis, we enrolled ten RCTs involving 1435 patients and pooled outcomes on EF and EJF after EEP vs TURP, including HoLEP $v s$ TURP, HoLEP vs PKRP (B-TURP), PKEP vs TURP, PKEP $v s$ PKRP (B-TURP), and ThuLEP $v s$ TURP. Among these RCTs, there were eight studies with results on EF at 6 to 92 months of follow-up, and we could notice a significant difference in IIEF-5 scores, which favors EEP at longer follow-up time ( $\geq 48$ months). On the contrary, the IIEF-5 score before 48 months of follow-up did not show statistically significant difference between EEP and TURP. This result suggests that EEP has the superiority in sustainable amelioration on patients' EF, when compared with TURP.

To avoid capsular perforation and severe venous bleeding, TURP is usually not extended up to the capsule fibers and the remnant of adenoma may be left during the resection of prostate. As a result, there may be a considerable recurrence rate in long-term follow-up after TURP. ${ }^{10} \mathrm{~A}$ second operation for recurrent $\mathrm{BPH}$, referred to as re-TURP, was reported to be performed at a constant annual rate of $\sim 1 \%-2 \%$ after TURP. ${ }^{4}$ In a large-scale analysis, the cumulative incidence of re-TURP for recurrence of prostatic hyperplasia was $2.9 \%$, $5.8 \%$, and $7.4 \%$, respectively, at 1,5 , and 8 years follow-up. ${ }^{32}$
In the procedure of EEP, removing adenoma of prostate along with the gouge of the surgical capsule could minimize the residual prostate tissue and reduce the chance of relapse of prostate tissue. In a recent review based on a prospectively collected database of a period of 18 years, HoLEP for LUTS/BPH was shown to offer a durable improvement in symptom and quality of life, with a reoperation rate as low as $1.4 \%(21 / 1476)$ in a median follow-up of 9.1 years. $^{33}$

ThuVEP also proved to be a durable procedure with regard to micturition improvement and prostate volume reduction with a low reoperation rate $(2.3 \%)$ during a 5-year followup. ${ }^{34}$ Similarly, PKEP was reported to have a high rate of adenoma clearance and to offer sustained improvements in micturition without recurrence during a long-term followup. ${ }^{10}$ Indeed, the RCTs with long-term results enrolled in our analysis unanimously showed that there no reoperation was required in the EEP arm, whereas there were several cases that required reoperation in TURP or PKRP arm. ${ }^{22,25,27}$

There were various energy sources for EEP applied in our analysis, but only one trial of thulium laser was enrolled, so the subgroup analyses were focused on the EF outcomes of HoLEP vs TURP and PKEP vs TURP, respectively. There was a significant difference favoring HoLEP with regard to IIEF-5 scores in long-term follow-up ( $\geq 48$ months). However, PKEP did not show this superiority to TURP, and no significant difference was found in IIEF-5 scores from 6- to 60-month evaluations in their subgroup. This result may be due to the discrepancy in details of the techniques between PKEP and HoLEP.

With similar safety and efficacy, PKEP was reported to require longer operative and recovery room times than HoLEP. ${ }^{35}$ The majority of PKEP is performed bluntly using the tip of the resectoscope or electrode, ${ }^{10}$ and this mechanical dissection will stretch the inner longitudinal layer around the apex of prostate, and might result in transient injury to the urethral sphincter complex and surrounding structures. ${ }^{36}$ Considering that the neurovascular bundle runs only $3 \mathrm{~mm}$ from the apical prostate capsule, and 3 to $4 \mathrm{~mm}$ from the membranous urethral lumen, ${ }^{37}$ further studies are needed to comprehensively evaluate the potential impact of mechanical enucleation on $\mathrm{EF}$.

Additionally, it is worth noting that in the RCT with longterm results enrolled in our analysis, ${ }^{25}$ the mean IIEF-5 was above 20 scores at preoperative baseline in both PKEP and TURP arms, and increased less than 3 scores after the surgery from 1 to 60 months follow-up. The considerable proportion of patients without preoperative ED weakened the positive effect of surgical treatments on sexual functions, and might conceal the potential advantage in persistent improvement of $\mathrm{EF}$ from one of these surgical approaches.

A variety of ejaculatory dysfunctions (EJD) appears in a considerable portion $(16.3 \%-55.7 \%)$ of patients with LUTS/BPH. ${ }^{38}$ Besides this, EJD is also the most common side effect complained by patients who undergo surgical treatment for LUTS/BPH, primarily in the form of RE. ${ }^{39}$ In a systematic review based on 30 RCTs, the incidence of RE in patients after TURP was $66.1 \%$, and there was no significant difference in EJD rates between standard monopolar resection and bipolar techniques. ${ }^{40}$ Although a few reports found an overall ejaculatory improvement, ${ }^{41,42}$ RE can make a substantial deleterious influence on the quality of life by reducing orgasmic intensity and inducing anxiety and depression. ${ }^{43}$ 
The pathophysiology of treatment-related RE has not been fully understood, but the predominant contributing factor was thought to be that the resection of the bladder neck and proximal portions of the prostate disrupts the internal urethral sphincter of maintaining antegrade ejaculatory function. ${ }^{44}$ Accordingly, it was taken for granted that the prostatic volume of removal is associated with the incidence of RE, and the EEP was assumed of more severe adverse effects to postoperative EJF compared with TURP. ${ }^{45}$

In our analysis, there were four RCTs that reported the incidence of RE after EEP and TURP, and no significant difference was found between the treatment arms. This result is in accordance with the findings of a systematic review ${ }^{40}$ and a meta-analysis ${ }^{15}$ with similar percentage of EJD among HoLEP and TURP groups. In addition, these studies assessed whether the incidence of RE is influenced by the volume of removed prostate tissue, and showed no significant difference in RE rate between the patients undergoing a standard TURP and those who accepted a minimal resection.

In the RCT enrolled in our analysis, PKEP had a comparable incidence of RE to TURP (59\% vs $62.8 \%$, respectively). ${ }^{21}$ Recently, a novel understanding of ejaculation physiology has enabled the emergence of modified surgical techniques of preserving antegrade ejaculation. Instead of the closure of the bladder neck, the structure around the verumontanum is considered to play an essential role in maintaining ejaculation in an antegrade fashion, even after the resection of bladder neck. ${ }^{46,47}$ This mechanism can give a reasonable explanation to the fact that EEP and TURP would deteriorate antegrade ejaculation in the same extent.

To our knowledge, this study is the first systematic review that includes a considerably large patient group and a longterm follow-up comparison to evaluate the postoperative EF and EJF between EEP and TURP for LUTS/BPH, with several strengths as following. First, this systematic review was designed with a precise focus based on a published protocol describing a comprehensive search strategy and a strict selection criteria for randomized clinical trials on the PROSPERO register (www.crd.york.ac.uk/PROSPERO). Second, this study included $10 \mathrm{RCTs}$ for final analysis, including 8 RCTs on EF and 4 on EJF, which could provide more outcomes and a full view on male sexual function after surgery. Third, compared with previous systematic reviews on this topic, the present study included more recent published trials especially one with a 72-month follow-up, which made it possible to evaluate the long-term effect of endoscopic enucleation on male sexual function.

We must admit some limitations in our meta-analysis that should be taken into consideration when interpreting the results. According to the EAU guidelines, ${ }^{4}$ we regarded EEP as a modality of ablation of prostatic tissue, which includes enucleations by both laser and bipolar energy systems. Therefore, we performed subgroup analyses to test for possible difference between HoLEP and PKEP. However, the statistical power was limited due to the relatively small sample size in the subgroup analysis. Besides this, the majority of patients in the eligible studies were followed with a period $<36$ months, leading to a scant number of studies available to evaluate long-term effect of intervention especially in the PKEP subgroup, which might influence the credibility of the results. Therefore, relevant findings should be viewed with caution. We are also unable to assess publi- cation bias using funnel plot given the limited number of studies, but our comprehensive search strategy, including unpublished studies, had lowered the relevant risk. Lack of blinding in several studies may introduce bias, but results were robust for the HoLEP subgroup with the exclusion of these studies.

\section{Conclusions}

Despite the lack of knowledge of the mechanisms of sexual changes after surgery for LUTS due to BPH, this systematic review demonstrates that the endoscopic enucleation has the superiority in sustainable preservation on EFs when compared with transurethral resection, although only a limited number of RCTs with long-term follow-ups are available. The subgroup analyses showed that the advantage of EEP was dominated by HoLEP at a long-term stage. In addition, no difference was evident regarding the rate of RE between EEP and TURP. Considering our existing limitations, more scientifically devised, prospective comparative studies with a large sample size and long-term follow-up should be undertaken to validate the difference on impact of sexual function after these two procedures.

\section{Author Contributions}

Y.L., Y.C., R.Z., J.L., and L.M. conceived and designed the study. Y.L. and Y.C. performed the database search, study selection, and data extraction. Y.C. and L.Z. conducted the statistical analysis. Y.L., Y.C., K.L., and C.X. drafted the article. L.Z., R.Z., J.L., and L.M. revised the article. Y.L. and Y.C. made equal contributions to this study. All authors read and approved the final article.

\section{Author Disclosure Statement}

No competing financial interests exist.

\section{Funding Information}

The study was sponsored by the National Natural Science Foundation of China [No. 61871004], the Fundamental Research Funds for Peking University [No. BMU2019 GJJXK009], and the Key Program on Clinical Subject of Peking University Third Hospital [No. BYSY2016020].

\section{Supplementary Material}

Supplementary Data

Supplementary Figure S1

Supplementary Figure S2

\section{References}

1. Vuichoud C, Loughlin KR. Benign prostatic hyperplasia: Epidemiology, economics and evaluation. Can J Urol 2015; 22 Suppl 1:1-6.

2. Spaliviero M, Strom KH, Gu X, et al. Does Greenlight HPS $^{\text {TM }}$ laser photoselective vaporization prostatectomy affect sexual function? J Endourol 2010;24:2051-2057.

3. Cindolo L, Pirozzi L, Fanizza C, et al. Drug adherence and clinical outcomes for patients under pharmacological therapy for lower urinary tract symptoms related to benign prostatic hyperplasia: Population-based cohort study. Eur Urol 2015;68:418-425. 
4. Gravas S, Cornu JN, Gacci M, et al. Guidelines on the management of non-neurogenic male lower urinary tract symptoms (LUTS). EAU Guidelines Office, Arnhem, The Netherlands. Available at: http://uroweb.org/guidelines/ compilations-of-all-guidelines (last accessed January 01, 2020).

5. Skolarikos A, Papachristou C, Athanasiadis G, et al. Eighteen-month results of a randomized prospective study comparing transurethral photoselective vaporization with transvesical open enucleation for prostatic adenomas greater than $80 \mathrm{cc}$. J Endourol 2008;22:2333-2340.

6. Kumar A, Vasudeva P, Kumar N, et al. A prospective randomized comparative study of monopolar and bipolar transurethral resection of the prostate and photoselective vaporization of the prostate in patients who present with benign prostatic obstruction: A single center experience. J Endourol 2013;27:1245-1253.

7. Kahokehr A, Gilling PJ. Enucleation techniques for benign prostate obstruction: Which one and why? Curr Opin Urol 2014;24:49-55.

8. Placer J, Salvador C, Planas J, et al. Effects of holmium laser enucleation of the prostate on sexual function. J Endourol 2015;29:332-339.

9. Tiburtius C, Knipper S, Gross AJ, et al. Impact of thulium VapoEnucleation of the prostate on erectile function: A prospective analysis of 72 patients at 12-month follow-up. Urology 2014;83:175-180.

10. Giulianelli R, Gentile BC, Mirabile G, et al. Bipolar plasma enucleation of the prostate: 5 years outcomes. J Endourol 2019;33:396-399.

11. Cornu JN, Ahyai S, Bachmann A, et al. A systematic review and meta-analysis of functional outcomes and complications following transurethral procedures for lower urinary tract symptoms resulting from benign prostatic obstruction: An update. Eur Urol 2015;67:1066-1096.

12. Sun F, Sun X, Shi Q, et al. Transurethral procedures in the treatment of benign prostatic hyperplasia: A systematic review and meta-analysis of effectiveness and complications. Medicine (Baltimore) 2018;97:e13360.

13. Zhang Y, Yuan P, Ma D, et al. Efficacy and safety of enucleation vs. resection of prostate for treatment of benign prostatic hyperplasia: A meta-analysis of randomized controlled trials. Prostate Cancer Prostatic Dis 2019;22:493-508.

14. Li Z, Chen P, Wang J, et al. The impact of surgical treatments for lower urinary tract symptoms/benign prostatic hyperplasia on male erectile function: A systematic review and network meta-analysis. Medicine (Baltimore) 2016;95: e3862.

15. Zong HT, Peng XX, Yang CC, et al. The impact of transurethral procedures for benign prostate hyperplasia on male sexual function: A meta-analysis. J Androl 2012;33:427-434.

16. Moher D, Liberati A, Tetzlaff J, et al. Preferred reporting items for systematic reviews and meta-analyses: The PRISMA statement. BMJ 2009;339:b2535.

17. Higgins JP, Altman DG, Gotzsche PC, et al. The Cochrane Collaboration's tool for assessing risk of bias in randomised trials. BMJ 2011;343:d5928.

18. Montorsi F, Naspro R, Salonia A, et al. Holmium laser enucleation versus transurethral resection of the prostate: Results from a 2-center, prospective, randomized trial in patients with obstructive benign prostatic hyperplasia. J Urol 2004;172:1926-1929.

19. Briganti A, Naspro R, Gallina A, et al. Impact on sexual function of holmium laser enucleation versus transurethral resection of the prostate: Results of a prospective, 2-center, randomized trial. J Urol 2006;175:1817-1821.

20. Wilson LC, Gilling PJ, Williams A, et al. A randomised trial comparing holmium laser enucleation versus transurethral resection in the treatment of prostates larger than 40 grams: Results at 2 years. Eur Urol 2006;50:569-573.

21. Zhao Z, Zeng G, Zhong W, et al. A prospective, randomised trial comparing plasmakinetic enucleation to standard transurethral resection of the prostate for symptomatic benign prostatic hyperplasia: Three-year follow-up results. Eur Urol 2010;58:752-758.

22. Gilling PJ, Wilson LC, King CJ, et al. Long-term results of a randomized trial comparing holmium laser enucleation of the prostate and transurethral resection of the prostate: Results at 7 years. BJU Int 2012;109:408-411.

23. Swiniarski PP, Stepien S, Dudzic W, et al. Thulium laser enucleation of the prostate (TmLEP) vs. transurethral resection of the prostate (TURP): Evaluation of early results. Cent European J Urol 2012;65:130-134.

24. Chen YB, Chen Q, Wang Z, et al. A prospective, randomized clinical trial comparing plasmakinetic resection of the prostate with holmium laser enucleation of the prostate based on a 2-year followup. J Urol 2013;189:217-222.

25. Zhu L, Chen S, Yang S, et al. Electrosurgical enucleation versus bipolar transurethral resection for prostates larger than $70 \mathrm{ml}$ : A prospective, randomized trial with 5-year followup. J Urol 2013;189:1427-1431.

26. Jhanwar A, Sinha R, Bansal A, et al. Outcomes of transurethral resection and holmium laser enucleation in more than $60 \mathrm{~g}$ of prostate: A prospective randomized study. Urol Ann 2017;9:45-50.

27. Gu M, Chen YB, Liu C, et al. Comparison of holmium laser enucleation and plasmakinetic resection of prostate: A randomized trial with 72-month follow-up. J Endourol 2018;32:139-143.

28. Liu Z, Li YW, Wu WR, et al. Long-term clinical efficacy and safety profile of transurethral resection of prostate versus plasmakinetic resection of the prostate for benign prostatic hyperplasia. Urology 2017;103:198-203.

29. Pavone C, Abbadessa D, Scaduto G, et al. Sexual dysfunctions after transurethral resection of the prostate (TURP): Evidence from a retrospective study on 264 patients. Arch Ital Urol Androl 2015;87:8-13.

30. Elshal AM, Elmansy HM, Elkoushy MA, et al. Male sexual function outcome after three laser prostate surgical techniques: A single center perspective. Urology 2012;80: 1098-1104.

31. Capogrosso P, Ventimiglia E, Ferrari M, et al. Long-term sexual outcomes after holmium laser enucleation of the prostate: Which patients could benefit the most? Int J Impot Res 2016;28:189-193.

32. Madersbacher S, Lackner J, Brossner C, et al. Reoperation, myocardial infarction and mortality after transurethral and open prostatectomy: A nation-wide, long-term analysis of 23,123 cases. Eur Urol 2005;47:499-504.

33. Ibrahim A, Alharbi M, Elhilali MM, et al. 18 Years of holmium laser enucleation of the prostate: A single center experience. J Urol 2019;202:795-800.

34. Gross AJ, Orywal AK, Becker B, et al. Five-year outcomes of thulium vapoenucleation of the prostate for symptomatic benign prostatic obstruction. World J Urol 2017;35:15851593.

35. Neill MG, Gilling PJ, Kennett KM, et al. Randomized trial comparing holmium laser enucleation of prostate with 
plasmakinetic enucleation of prostate for treatment of benign prostatic hyperplasia. Urology 2006;68:1020-1024.

36. Hirasawa Y, Kato Y, Fujita K. Age and prostate volume are risk factors for transient urinary incontinence after transurethral enucleation with bipolar for benign prostatic hyperplasia. Int J Urol 2018;25:76-80.

37. Ponnusamy K, Sorger JM, Mohr C. Nerve mapping for prostatectomies: Novel technologies under development. J Endourol 2012;26:769-777.

38. Cho MC, Kim JK, Song SH, et al. Patient-reported ejaculatory function and satisfaction in men with lower urinary tract symptoms/benign prostatic hyperplasia. Asian J Androl 2018;20:69-74.

39. Welliver C, Essa A. Sexual side effects of medical and surgical benign prostatic hyperplasia treatments. Urol Clin North Am 2016;43:393-404.

40. Marra G, Sturch P, Oderda M, et al. Systematic review of lower urinary tract symptoms/benign prostatic hyperplasia surgical treatments on men's ejaculatory function: Time for a bespoke approach? Int J Urol 2016;23:22-35.

41. Muntener M, Aellig S, Kuettel R, et al. Sexual function after transurethral resection of the prostate (TURP): Results of an independent prospective multicentre assessment of outcome. Eur Urol 2007;52:510-515.

42. Brookes ST, Donovan JL, Peters TJ, et al. Sexual dysfunction in men after treatment for lower urinary tract symptoms: Evidence from randomised controlled trial. BMJ 2002;324:1059-1061.

43. Lebdai S, Chevrot A, Doizi S, et al. Do patients have to choose between ejaculation and miction? A systematic review about ejaculation preservation technics for benign prostatic obstruction surgical treatment. World J Urol 2019; 37:299-308.

44. Borchert A, Leavitt DA. A review of male sexual health and dysfunction following surgical treatment for benign prostatic hyperplasia and lower urinary tract symptoms. Curr Urol Rep 2018;19:66.

45. $\mathrm{Xu} \mathrm{P}, \mathrm{Xu} \mathrm{A}, \mathrm{Chen} \mathrm{B}$, et al. Bipolar transurethral enucleation and resection of the prostate: Whether it is ready to supersede TURP? Asian J Urol 2018;5:48-54.

46. Alloussi SH, Lang C, Eichel R, et al. Ejaculationpreserving transurethral resection of prostate and bladder neck: Short- and long-term results of a new innovative resection technique. J Endourol 2014;28:84-89.

47. Kim M, Song SH, Ku JH, et al. Pilot study of the clinical efficacy of ejaculatory hood sparing technique for ejaculation preservation in Holmium laser enucleation of the prostate. Int J Impot Res 2015;27:20-24.
Address correspondence to:

Jian $\mathrm{Lu}, \mathrm{MD}$

Department of Urology

Peking University Third Hospital

No. 49, North Garden Road

Haidian District

Beijing 100191

China

E-mail: lujian@bjmu.edu.cn

Rongsheng Zhao, PhD

Department of Pharmacy

Peking University Third Hospital

No. 49, North Garden Road

Haidian District

Beijing 100191

China

E-mail: zhao_rongsheng@163.com

$\begin{aligned} \text { Abbreviations Used } & \\ \mathrm{BPH} & =\text { benign prostatic hyperplasia } \\ \mathrm{B}-\mathrm{TURP} & =\text { bipolar transurethral resection of the prostate } \\ \mathrm{CI} & =\text { confidence interval } \\ \mathrm{EAU} & =\text { European Association of Urology } \\ \mathrm{ED} & =\text { erectile dysfunction } \\ \mathrm{EEP} & =\text { endoscopic enucleation of the prostate } \\ \mathrm{EF} & =\text { erectile function } \\ \mathrm{EJD} & =\text { ejaculatory dysfunctions } \\ \mathrm{EJF} & =\text { ejaculation function } \\ \mathrm{HoLEP} & =\text { holmium laser enucleation of the prostate } \\ \mathrm{IIEF}-5 & =\text { International Index of Erectile Function } 5 \\ \mathrm{LUTS} & =\text { lower urinary tract symptoms } \\ \mathrm{MD} & =\text { mean difference } \\ \mathrm{PKEP} & =\text { plasmakinetic enucleation of the prostate } \\ \mathrm{PKRP} & =\text { plasmakinetic resection of prostate } \\ \mathrm{PRISMA} & =\text { Preferred Reporting Items for Systematic } \\ & \text { Reviews and Meta-Analyses } \\ \mathrm{RCT} & =\text { randomized controlled trial } \\ \mathrm{RE} & =\text { retrograde ejaculation } \\ \mathrm{RR} & =\text { risk ratios } \\ \text { ThuLEP } & =\text { thulium laser enucleation of the prostate } \\ \text { ThuVEP } & =\text { thulium vapoenucleation of the prostate } \\ \mathrm{TURP} & =\text { transurethral resection of the prostate } \\ & \end{aligned}$

